\title{
A Novel Method of Mechanical Oxidation of CNT for Polymer Nanocomposite Application: Evaluation of Mechanical, Dynamic Mechanical, and Rheological Properties
}

\author{
Priyanka Pandey, Smita Mohanty, and Sanjay Kumar Nayak \\ Central Institute of Plastics Engineering and Technology (CIPET), T.V.K Industrial Estate, Guindy, Chennai 600032, India \\ Correspondence should be addressed to Sanjay Kumar Nayak; papers.journal@gmail.com
}

Received 16 January 2014; Accepted 30 March 2014; Published 17 April 2014

Academic Editor: Donald L. Feke

Copyright (C) 2014 Priyanka Pandey et al. This is an open access article distributed under the Creative Commons Attribution License, which permits unrestricted use, distribution, and reproduction in any medium, provided the original work is properly cited.

\begin{abstract}
A new approach of oxidation of carbon nanotubes has been used to oxidize the CNTs. A comparative aspect of the mechanical oxidation and acid oxidation process has been established. FTIR analysis and titration method have shown the higher feasibility of the mechanical oxidation method to oxidize the CNTs. Comparatively less damage to the CNTs has been observed in case of mechanically oxidized as compared to acid oxidized CNTs. The mechanical properties of the nanocomposites reinforced with the acid oxidized CNT (ACNT) and mechanically oxidized CNTs (McCNT) were analyzed and relatively higher properties in the nanocomposites reinforced with McCNT were noticed. The less degree of entanglement in the McCNTs was noticed as compared to ACNTs. The dynamic mechanical analysis of the nanocomposites revealed much improved load transfer capability in the McCNT reinforced composites. Further, the rheological properties of the nanocomposites revealed the higher performance of McCNT reinforced composites.
\end{abstract}

\section{Introduction}

Tremendously high mechanical properties with low density of multiwalled carbon nanotubes (MWNT) have prompted investigation of these nanomaterials as filler in polymer matrix [1-3]. The properties of MWNTs depend strongly on the dispersion of the nanotubes inside polymer matrix. However, the hydrophobic inert nature of nanotubes causes bundling of tubes [4]. The bundling of nanotubes can be minimized by modification of surface properties of tubes [5]. Oxidation method is the most efficient method in this regard $[6,7]$. Further, acids have been extensively utilized for this purpose. However, the acid oxidation of nanotubes has exhibited damage to the nanotube surface. Hence, this study was aimed to establish an oxidation method, to improve the dispersibility of nanotubes, with minimized damage to the nanotube surface and its crystalline organization.

The novel method used in this study to minimize the damage was based on the mechanical oxidation of the nanotubes $[8,9]$. The confirmation of modification was carried out via Fourier transform infrared spectroscopic (FTIR) analysis. Raman spectra were analyzed to evaluate the effect of both modifications on CNT properties. The concentration of acidic groups on the modified (oxidized) CNTs was determined using titration method. The TEM micrographs have been analyzed in order to investigate the effect of both the oxidation methods on the surface characteristics of CNT and its bundling behavior. Further, in order to evaluate the effect of this oxidation method on the polymer matrix, polymer nanocomposites were prepared and an investigation of the properties of the polymer nanocomposites was carried out. Polypropylene has been used as the matrix materials, since it has acquired a large space in the mainstream industry like automobile, packaging, and so forth [10]. The major requirement of the nanocomposites is the good mechanical property [11] and hence a study on effect of these modifications on the mechanical properties was carried out. Further, the dynamic mechanical analysis technique was used to analyze the thermomechanical performance of the nanocomposites 
and a comparative aspect of the effect of the mechanical oxidation as compared to acid oxidation was established. Further, the rheological property of the nanocomposites was investigated to analyze the effect of this novel approach of modification on the microstructural characteristics of the nanocomposites. This was further supported by TEM micrographs of nanocomposites.

\section{Experimental}

2.1. Oxidation of Carbon Nanotubes (CNTs). In a typical procedure, $2 \mathrm{mg}$ of CNT sample was refluxed in $50 \mathrm{~mL}$ of 2.6 $\mathrm{M} \mathrm{HNO}_{3}$, for $48 \mathrm{hrs}$. The mixture was diluted in $500 \mathrm{~mL}$ of distilled water and functionalized CNT was collected through filtration. This type of treated CNT is termed ACNT for further discussion.

A mixture of $0.4 \mathrm{~g}$ of $\mathrm{CNT}$ and $8 \mathrm{gm}$ of potassium hydroxide and ethanol was stirred manually and the mixture was allowed for intensive ball milling for $3 \mathrm{hrs}$, using a ball milling machine. The obtained mixture was dissolved in deionized water and centrifuged. This dissolution and centrifugation process was repeated to ensure the complete removal of $\mathrm{KOH}$ residues. The collected samples were dried for $10 \mathrm{hrs}$ at $100^{\circ} \mathrm{C}$. This type of treated CNT is termed $\mathrm{McCNT}$ for further discussion.

2.2. Fabrication of Nanocomposites. The masterbatch route was employed to prepare the nanocomposites using microinjection molding technique. First the PP/MAPP hybrid was synthesized with a $5 \mathrm{wt} \%$ loading of MAPP using compounder, M/s DSM explore Netherlands, Micro 15 at a temperature of $180^{\circ} \mathrm{C}$, for 10 minutes as obtained from previous study. In the second step polypropylene (PP) nanocomposites were prepared with 0.3 weight \% loading of ACNT and $\mathrm{McCNT}$ at a temperature of $180^{\circ} \mathrm{C}, 185^{\circ} \mathrm{C}$, and $180^{\circ} \mathrm{C}$ in front, middle, and rear zone, respectively.

2.3. Fourier Transform Infrared Spectroscopy (FTIR). For the confirmation of successful modification of Na-MMT the Fourier transform infrared spectroscopy (FTIR) was carried out using thermoscientific FTIR (smart orbit ATR 400$4000 \mathrm{~cm}^{-1}$ with microscope). The heat dried samples of treated clays have been used for analysis.

2.4. Raman Spectroscopy. Raman scattering spectra were recorded by Micro-Raman LabRam system in a backscattering geometry. A $632.8 \mathrm{~nm}(1.96 \mathrm{eV}) \mathrm{HeNe}$ laser was used as the light source and the power of the laser was adjusted by optical filters. The typical acquisition time for the spectra was $30 \mathrm{sec}$.

2.5. Transmission Electron Microscope (TEM). TEM analysis was of the samples carried using transmission electron microscope (JEOL 1200EX, Japan). TEM imaging was carried out at an accelerating voltage of $100 \mathrm{kV}$. Images were captured using a charged couple detector (CCD) camera for further analysis using Gatan Digital Micrograph analysis software.
2.6. Mechanical Properties. The Izod impact strength of the $\mathrm{PP}$ and its nanocomposites have been evaluated using Tinius Olsen, USA, impactometer as per ASTM D 256. The samples were notched prior to the testing prior to loading with hammer of $2 \mathrm{~J}$. The notch depth was taken as $2.53 \mathrm{~mm}$ with a notch angle of $45^{\circ}$. The microinjection molded samples of diameter $63.5 \times 12.7 \times 3 \mathrm{~mm}^{3}$ have been used for the testing. 7 numbers of samples of each composition have been tested in order to get the accuracy in the result.

Further, the microinjection molded dog-bone shaped samples ( 5 samples of each composition) have been subjected to tensile test. Tensile properties of the virgin matrix as well as the nanocomposites have been evaluated using Universal Tensile Machine (3382 Instron, UK) as per ASTM D 638. Samples of $127 \times 12.7 \times 3 \mathrm{~mm}^{3}$ dimension were subjected to tensile test at a gauge length of $50 \mathrm{~mm}$ and with crosshead speed of $5 \mathrm{~mm} / \mathrm{min}$.

2.7. Dynamic Mechanical Analysis (DMA). The samples were tested using dynamic mechanical analyzer (TA Instruments Q800) to determine the effects of modifications on the dispersion of clay in the nanocomposites. The samples were tested at $-100^{\circ} \mathrm{C}$ to $150^{\circ} \mathrm{C}$ to obtain information across transition temperature. The DMA analysis was conducted in a dual cantilever mode. The experiment was performed with the injection molded samples having a dimension of $63.5 \times$ $12.7 \times 3 \mathrm{~mm}^{3}$.

2.8. Rheological Analysis. The samples were characterized by a modular advanced rheometer system (MARS III, Thermo Fisher Scientific, Germany) in the frequency sweep mode using parallel plate fixtures of $25 \mathrm{~mm}$ in diameter at a gap of $1 \mathrm{~mm}$. In order to investigate the formation of silicate network and extensive morphology frequency sweep method is most often used methodology. Hence, the dynamic frequency sweep test was performed at $220^{\circ} \mathrm{C}$ and frequency sweep from $0.1 \mathrm{rad} / \mathrm{sec}$ to $200 \mathrm{rad} / \mathrm{sec}$ at a constant strain amplitude of $1 \%$. Corresponding storage modulus $\left(G^{\prime}\right)$, loss modulus $\left(G^{\prime \prime}\right)$, and complex viscosity $\left(\eta^{*}\right)$ were determined as a function of frequency and the data were analyzed and represented.

\section{Materials}

Polypropylene (M110) was procured from M/s Haldia Petrochemicals, Kolkata, India, having a density of $0.94 \mathrm{~g} / \mathrm{cm}^{3}$ and the MFI of $11 \mathrm{~g} / 10 \mathrm{~min}$. Multiwalled carbon nanotubes of $>98 \%$ purity and diameter of $80-100 \mathrm{~nm}$, used in this work, was purchased from M/s Nanoshel, Intelligent Materials Pvt. Ltd, India. The compatibilizer used in this study was maleic anhydride grafted polypropylene (MAPP), (OPTIMP425) having an anhydride content of 1.6-2.5\% and density $0.91 \mathrm{~g} / \mathrm{cm}^{3}$ was purchased from M/s Pluss Polymers Pvt. Ltd., Haryana, India.

\section{Results and Discussion}

4.1. Analytical Characterization through Titration Method. The total acidic sites were quantified by titration method. 


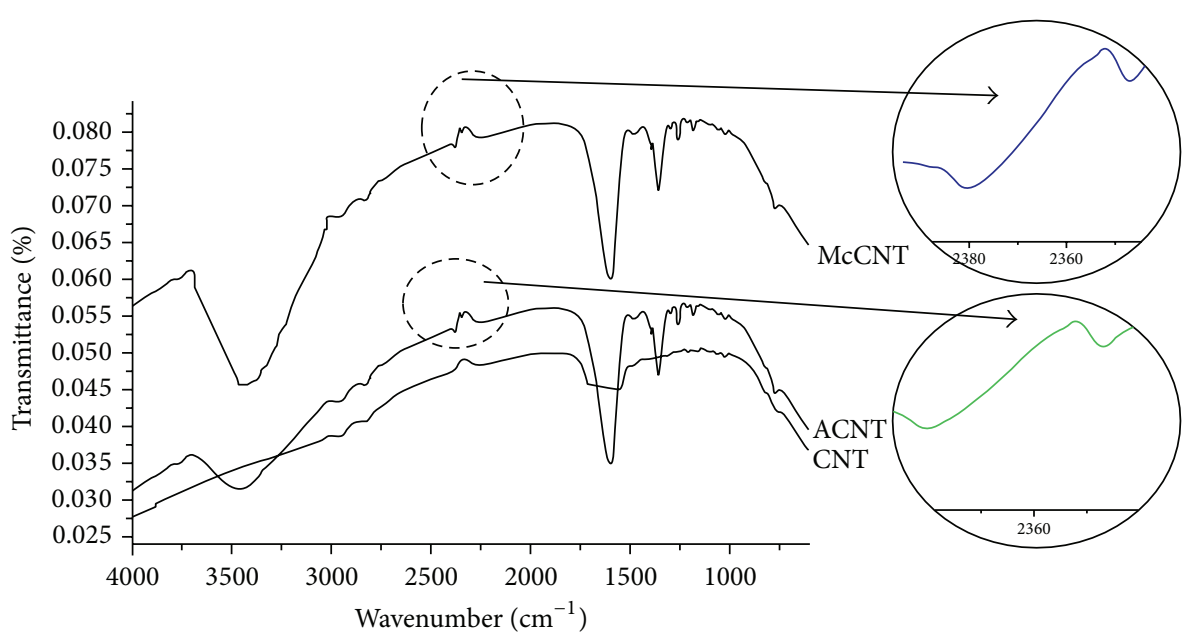

FIGURE 1: FTIR spectrum of pristine and treated CNTs.

First $0.2 \mathrm{mg}$ of treated CNTs was sonicated in $2.5 \mathrm{~mL}$ of $0.1 \mathrm{M}$ $\mathrm{NaOH}$ and the mixture was kept for stirring for $6 \mathrm{hrs}$ under inert atmosphere. The precipitation of CNTs was carried out by adding $\mathrm{CaCl}_{2}$. Precipitate was separated from the solution and removed upon washing. Further, $2.5 \mathrm{~mL}$ of $\mathrm{HCl}$ was added to the filtrate with constant stirring for stirring under inert atmosphere for $12 \mathrm{hrs}$. Excess of $\mathrm{HCl}$ was determined by titration with $\mathrm{NaOH}$ [12]. The relative concentration of the acidic group was found to be $1.7,3.4$, and $4.6 \mathrm{mmol} / \mathrm{g}$ for $\mathrm{CNT}$, ACNT, and McCNT, respectively. It was evident that mechanical oxidation process results in higher concentration of acidic sites as compared to that of acid treatment.

4.2. Fourier Transform Infrared Spectroscopy (FTIR). Figure 1 represents the FTIR spectra of as received CNT and oxidized CNTs (i.e., ACNT and McCNT). A prominent absorption band at $1574 \mathrm{~cm}^{-1}$, in the spectra of as received CNT, is related to the carbon skeleton of the nanotubes. In case of McCNT this peak was shifted to $1599 \mathrm{~cm}^{-1}$, respectively, indicating the increased density of surface oxygen [13]. In case of both the treated CNTs (i.e., ACNT and McCNT), additional peaks near $1084 \mathrm{~cm}^{-1}$ and $1406 \mathrm{~cm}^{-1}$ were observed, exhibiting the $\mathrm{C}-\mathrm{O}-\mathrm{C}$ stretching group (from ether, alcohol, and carboxylic acid) and characteristic hydroxyl bond $(\mathrm{O}-\mathrm{H})$. A broad absorption band near $3434 \mathrm{~cm}^{-1}$ (H-bond in hydroxyl, carboxylic, and phenol groups) was also present in both oxidized CNTs (i.e., ACNT and McCNT). Therefore, form the FTIR analysis it was found that oxygenated functional groups have been introduced onto the surface of CNTs, revealing the confirmation of oxidation.

Hence, it is worth mentioning that both the modification processes are feasible to incorporate the surface acidic group in the CNTs. The comparative investigation of the oxidation efficiency of both the methods is discussed in the further study.

4.3. Raman Spectroscopy. Figure 2 represents the Raman spectra of CNTs excited with the $514.5 \mathrm{~nm}$ laser line. All the samples exhibited three characteristic bands, namely, D-band

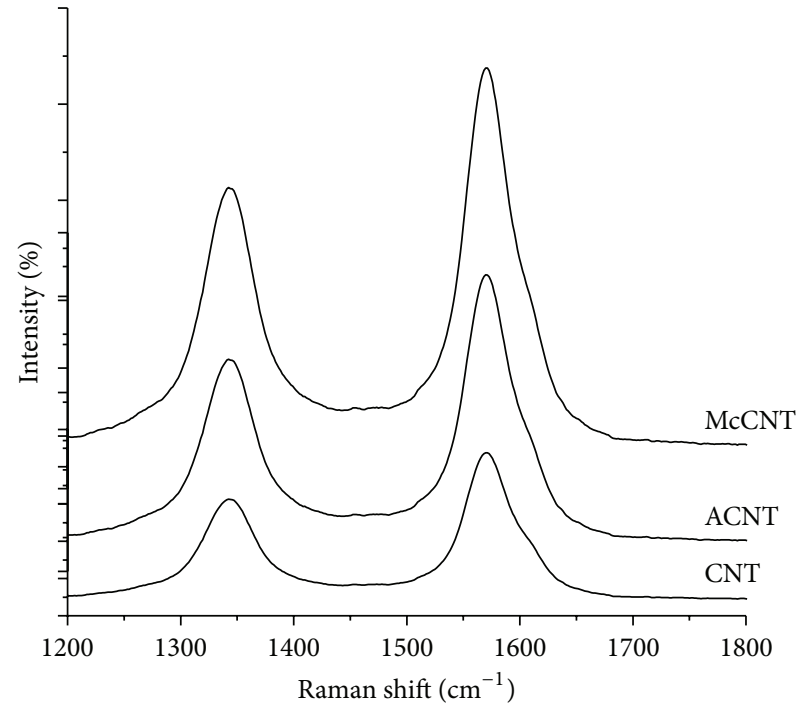

FIGURE 2: Raman spectrum of pristine and oxidized CNTs.

$\sim 1338 \mathrm{~cm}^{-1}$, G-band $\sim 1572 \mathrm{~cm}^{-1}$, and $\mathrm{D}^{\prime}-1608 \mathrm{~cm}^{-1}[9,14]$. The $\mathrm{D}$-band is a disorder induced carbon atoms resulting from the defects in the CNT $[15,16]$. However, G-band relates to the structural intensity of $\mathrm{sp}^{2}$ hybridized carbon atoms of CNT [17].

In case of treated CNTs higher D-band intensity was noticed as compared to neat CNT, confirming the conversion of $\mathrm{sp}^{2}$ hybridization to the $\mathrm{sp}^{3}$ carbon. This effect was more pronounced in McCNT, revealing the transformation of relatively higher number of $\mathrm{sp}^{2}$ carbon atoms into $\mathrm{sp}^{3}$ carbon. Further, higher ratio of relative intensity of G-band to D-band $\left(I_{G} / I_{D}\right)$ of neat CNT as compared to treated CNTs exhibited the increase in the degree of disorderness and presence of the defects on the surface of treated CNT, arising from the foreign functionalities of the tube surface. Further, the lower relative intensity $\left(I_{G} / I_{D}\right)$ of the McCNT as compared to that of ACNT revealed the higher number of defects on the McCNT 


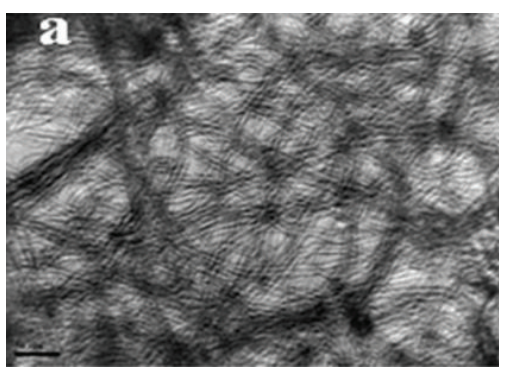

(a)

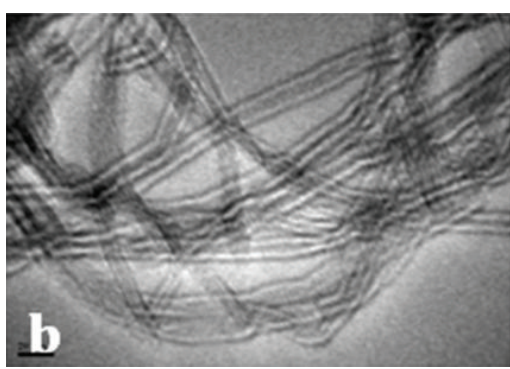

(b)

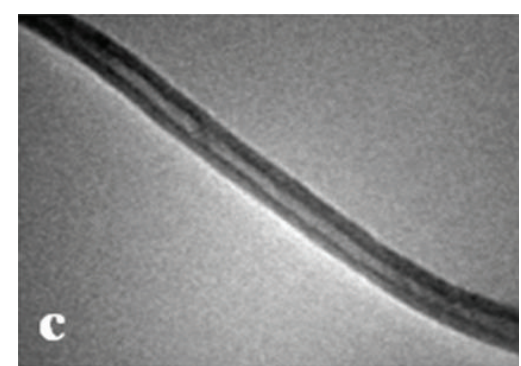

(c)

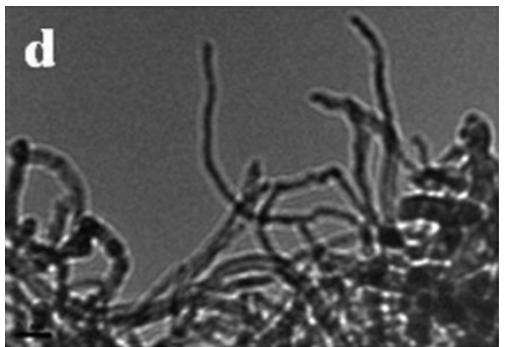

(d)

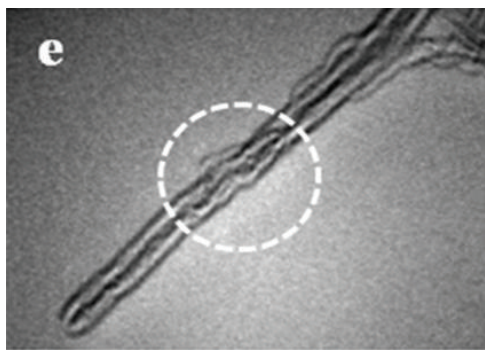

(e)

FIgUre 3: TEM micrographs of (a) pristine CNT; (b) and (c) McCNT; (d) and (e) ACNT.

as compared to ACNT [18]. Also the lower $\left[I_{G} /\left(I_{G}+I_{D}\right)\right]$ values (i.e., qualify factor) of $\mathrm{McCNT}$ as compared to that of ACNT indicate the less cutting of the CNT length during mechanochemicial oxidation treatment at applied conditions [19].

4.4. Transmission Electron Microscope (TEM). The surface of the CNTs was analyzed under TEM and reported in Figures 3(a)-3(e). The dispersed CNT samples were analyzed. TEM micrographs of neat CNT exhibited various bundles. However, individual fibers were visible in case of both treated CNTs. However, in case of McCNT the bundling was found to be minimized as compared to ACNT. This may result in the relatively better dispersion of $\mathrm{McCNT}$ in polymer matrix. Further, the damage in the surface of ACNT, as observed from previous characterization, was also confirmed from Figure 3(c). However, no sign of surface damage could be seen in McCNT (Figure 3(e)).

4.5. Mechanical Properties. Table 1 represents the mechanical properties of the PP and its nanocomposites. The mechanical properties were evaluated through analyzing their tensile and impact properties and represented the injection molded sample. In order to maintain the accuracy of results five samples of each composition were tested. Ductile fracture of the samples was noticed in all materials. The mechanical properties of PP/MAPP/ACNT and PP/MAPP/McCNT were found considerably higher than that of neat PP. This is attributed to the stress transfer ability in carbon nanotube polymer composites [20] due to the tendency of nanotubes to align and bridge the crack when a tensile stress is applied to the composites leading to load transfer across the polymer nanotube interface. Wherein, relatively higher tensile properties of PP/MAPP/McCNT revealed much efficient load transfer properties between McCNT and matrix polymer [20] as compared to that of ACNT.

This is ascribed to the relatively more even dispersion of McCNTs in the polymer matrix arising from less disentangled CNT fibrils, as was noticed in TEM micrographs. This further leads to more uniform bridging of crack. This also supports the fact that ACNT reinforced nanocomposites, due to damaged structural integrity, may have some loose interaction leading to the relatively nonuniform stress transfer across polymer nanotube interface unlike McCNT reinforced nanocomposites.

4.6. Dynamic Mechanical Analysis. In order to confirm the finding of filler dispersion as obtained from mechanical analysis, the dynamic mechanical analysis is the preferred method, wherein the storage modulus is related to the stiffness of the material and measures the elastic response of the polymer. The loss modulus denotes the energy dissipated by the system in the form of heat and measures the viscous response of the polymer material, which in turn provides the information about the mechanical properties of the material. The damping factor $(\tan \delta)$ is the ratio of the loss modulus to storage modulus and helps in estimating filler-polymer interaction in case of composites.

Figures 4(a) and 4(b) represent a variation of storage modulus and loss modulus with respect to temperature. In PP over the entire temperature range, two main mechanical relaxation processes were evident, namely, high temp $\alpha$ relaxation, related to the crystalline fraction present, and a $\beta$ process, related to the glass/rubber transition relaxation. A general falling trend was observed in all the cases.

The higher initial value of storage modulus was observed for each sample at a subambient temperature. This supports 
TABLE 1: Mechanical properties of PP and PP nanocomposites.

\begin{tabular}{lcccc}
\hline Composition & Yield strength $(\mathrm{MPa})$ & Young's modulus $(\mathrm{MPa})$ & Elongation at Break $(\%)$ & Impact strength $(\mathrm{j} / \mathrm{m})$ \\
\hline PP/MAPP & $30.71 \pm 0.18$ & $1193 \pm 32$ & $>500$ & 37 \\
PP/MAPP/ACNT & $35.98 \pm 0.27$ & $1630 \pm 60$ & $>500$ & 47 \\
PP/MAPP/McCNT & $36.33 \pm 0.41$ & $1727 \pm 57$ & $>500$ & 51 \\
\hline
\end{tabular}

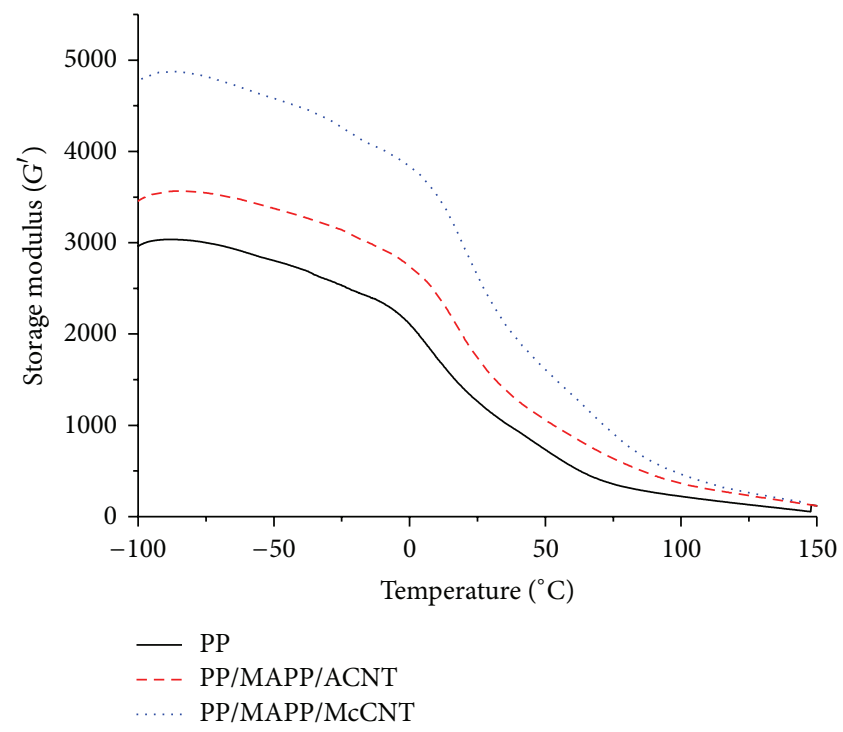

(a)

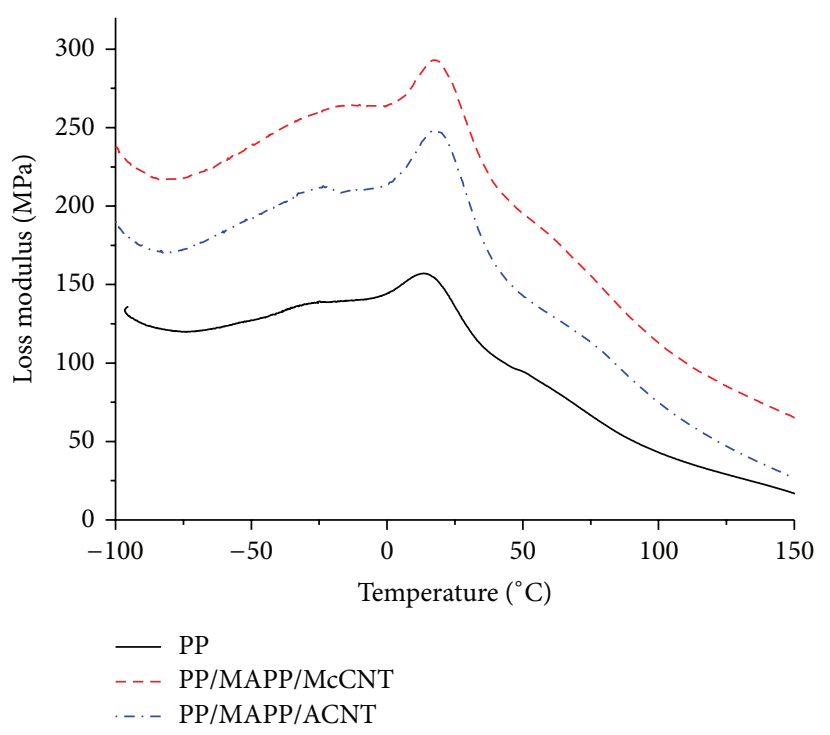

(b)

FIGURE 4: Storage modulus and loss modulus of PP and its nanocomposites.

the fact that molecules remain in a frozen state in this condition, and hence they show high stiffness properties in glassy condition. A clear transition was observed at $0^{\circ} \mathrm{C}$. This transition might be related to the glass $(\beta)$ transition. In all the cases, it was found that the storage modulus value decreases with the increase in temperature, below glass transition temperature. This might be due to the fact that PP reaches its softening point and therefore reduces the elastic response of the material. A considerable drop was noticed in the vicinity of glass transition temperature, indicating the phase transition from the rigid glassy state where the molecular motions are restricted to a more flexible rubbery state and the molecular chains have more freedom to move. Further, with the increase of temperature to the melting temperature the storage modulus of composites is dominated by matrix intrinsic modulus. Storage modulus is higher when the molecular movement is limited or restricted and it consequently will cause the storage of mechanical energy to increase [21]. The stiffening effect was more remarkable at lower temperature. This phenomenon was explained by the mismatch in coefficient of thermal expansion between the matrix and inorganic fillers, which might allow better stress transfer between matrices and fillers at low temperatures [22]. The similar observation was found in all cases of composites also.

Further, it was noticed that the storage modulus of the composites was higher as compared to the PP. This confirmed the reinforcing effect of ACNT and McCNT in their individual composites. Subsequently, higher storage modulus of $\mathrm{PP} / \mathrm{MAPP} / \mathrm{McCNT}$ composites as compared to $\mathrm{PP} / \mathrm{MAPP} / \mathrm{ACNT}$ revealed more evenly dispersed McCNT particles in the PP matrix, leading to the relatively more even distribution of the stress [23]. This, further, exhibited the higher surface area of McCNT particles in PPg matrix, arising from the relatively finer dispersion of McCNT inside the PPg matrix [23]. This further leads to the much more evenly transferred applied stresses from matrix onto the CNT particles.

The $\beta$ relaxation, related to the local motion of amorphous phase, corresponding to glass transition temperature $\left(t_{g}\right)$ of PP was observed at $17^{\circ} \mathrm{C}$ and no further change could be noticed in that of composites, revealing the equal level of crystallinity of PP and its composites. Hence, it is evident that incorporation of CNT and any type of treated CNTs did not alter the relaxation mechanism of macromolecular chains. This may be attributed to the rapid crystallization of the polymer and thus the anticipated effect of reinforcement is masked.

Figure 5 represents the damping factor of $\mathrm{PP}$ and its nanocomposites. The damping in the polymeric material is sensitive to segmental mobility of the polymer chains and in composites is the indicative of interfacial interaction between the polymer and the filler. Strong interfacial interaction between the polymer and the filler tends to restrict the polymer mobility thereby reducing the damping, wherein the lowest damping factor of PP/MAPP/McCNT as compared to 


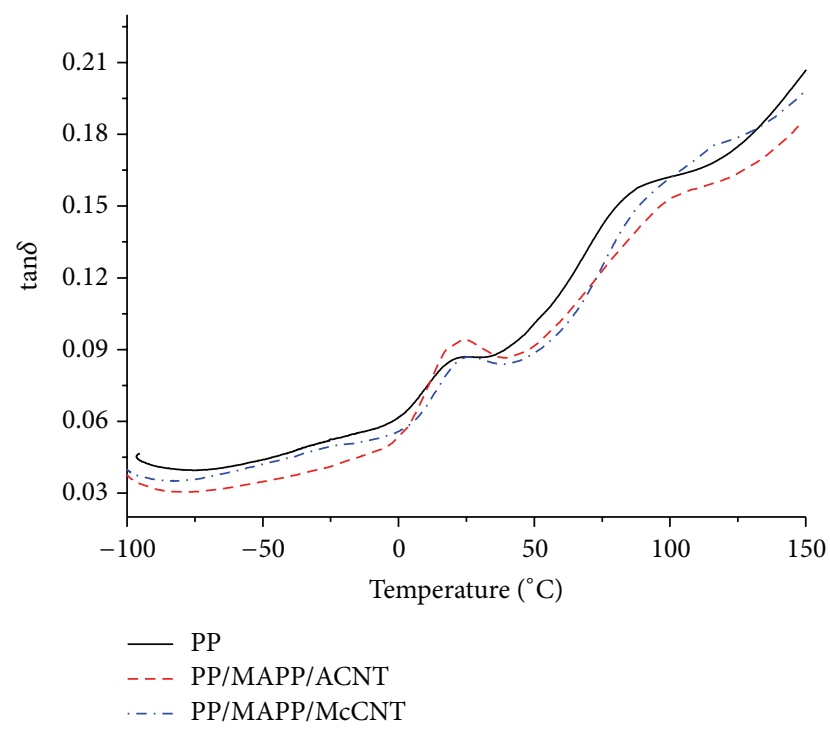

FIGURE 5: Tan $\delta$ versus temperature of PP and its nanocomposites.

$\mathrm{PP} / \mathrm{MAPP} / \mathrm{ACNT}$ revealed the relatively strong interaction between McCNT and polymer matrix, causing the restricted polymer mobility.

4.7. Rheological Assessment. The rheological properties can provide information about the percolated network structure as well as the interaction between filler and polymer matrix. Moreover, it is important to evaluate the rheological behavior in order to understand the effect of the nanotubes on internal structures and processing properties of polymer nanocomposites [24].

Figure 6 and Table 2 represent the complex viscosity of $\mathrm{PP}$ and its nanocomposites as a function of frequency. From the figure it was depicted that the complex viscosities $\left(\eta^{*}\right)$ of $\mathrm{PP}$ and its nanocomposites were decreased with increasing frequency, indicating a non-Newtonian behaviour over the whole frequency range measured.

The shear thinning effect, noticed in case of nanocomposites, was ascribed to the random orientation and entangled molecular chains in the nanocomposites during the applied shear force. The PP nanocomposites containing McCNT and ACNT nanocomposites exhibited higher $\eta^{*}$ value than that of neat PP at low frequency, indicating the interconnected and network structures formed as a result of particleparticle and particle-polymer interactions. Further, both the nanocomposites exhibited shear thinning behavior, revealing the breakdown of these interactions and network structures with increase in the applied frequency. Subsequently, the $\eta^{*}$ of the PP/MAPP/McCNT nanocomposites was found to be relatively higher as compared to $\mathrm{PP} / \mathrm{MAPP} / \mathrm{ACNT}$, revealing the strong interaction between McCNT and polymer as compared to that of ACNT and Polymer. Furthermore, relatively more distinct shear thinning behaviour and $\eta^{*}$ over the whole applied frequency range, as compared to PP/MAPP/ACNT, might be ascribed to better dispersion of McCNT and strong ACNT polymer interaction [14-25].

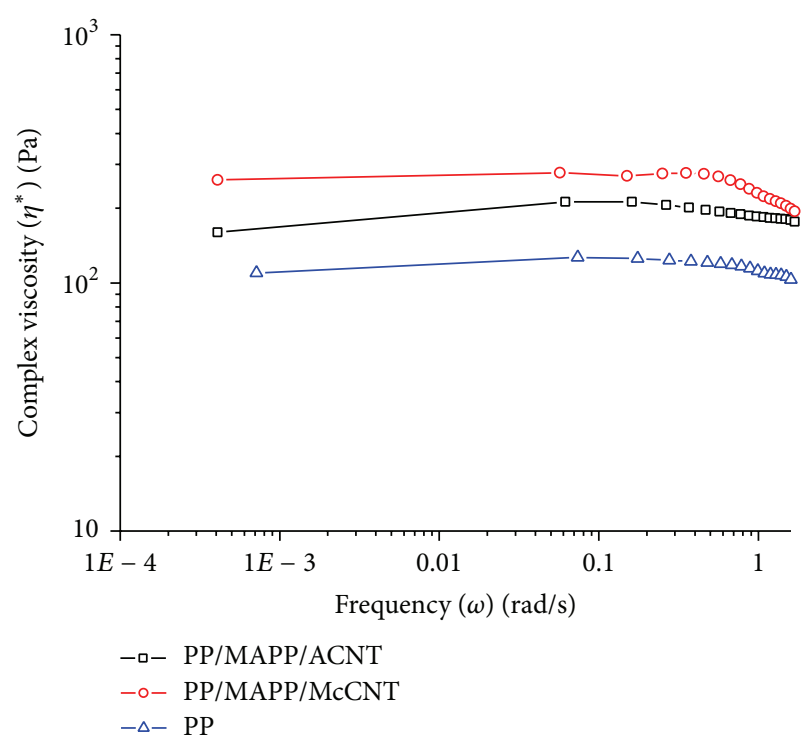

FIGURE 6: Complex viscosity versus frequency of PP and nanocomposites.

TABLE 2: Variations of low frequency slopes of $\eta^{*}, G^{\prime}$, and $G^{\prime \prime}$ versus $\omega$ for PP and its nanocomposites.

\begin{tabular}{lccc}
\hline Materials & $\begin{array}{r}\text { Slope of } \eta^{*} \\
\text { versus } \omega\end{array}$ & $\begin{array}{c}\text { Slope of } G^{\prime} \\
\text { versus } \omega\end{array}$ & $\begin{array}{r}\text { Slope of } G^{\prime \prime} \\
\text { versus } \omega\end{array}$ \\
\hline PP & -0.11 & 1.21 & 0.97 \\
PP/MAPP/McCNT & -0.14 & 1.05 & 0.91 \\
PP/MAPP/ACNT & -0.16 & 0.87 & 0.89 \\
\hline
\end{tabular}

Further, the shear thinning exponent $(n)$ for the nanocomposites was determined using

$$
\left|\eta \cdot * \approx \omega^{n}\right|
$$

From the analysis it was depicted that shear thinning behavior of the nanocomposites was dependent on the presence of McCNT and ACNT. The incorporation of McCNT and ACNT resulted in decreased value of " $n$ " and this effect was more pronounced in case of $\mathrm{PP} / \mathrm{MAPP} / \mathrm{McCNT}$. This can be again ascribed to the enhanced interfacial interaction between ACNT and polymer matrix. The difference in the $n$ value of both the nanocomposites depicted the variable efficiency of the modification. Hence, it is worth mentioning that the McCNT is relatively better reinforcing agent for PP matrix in presence of MAPP [14].

Figure 7 represents the frequency dependence of the storage modulus $\left(G^{\prime \prime}\right)$ and loss modulus $\left(G^{\prime \prime}\right)$ for the PP and its nanocomposites measured at $220^{\circ} \mathrm{C}$. The value of storage modulus $\left(G^{\prime}\right)$ and loss modulus $\left(G^{\prime \prime}\right)$ of the PP and its nanocomposites showed an increasing tendency with increase in the frequency. This effect was more significant in the low frequency region, revealing the relaxation behavior of polymer to a long time scale, in the low frequency region [14]. At low frequencies, frequency dependence of modulus weakens clearly with the addition of the CNTs, indicating that the long-range motion of the polymer chains is restrained by 


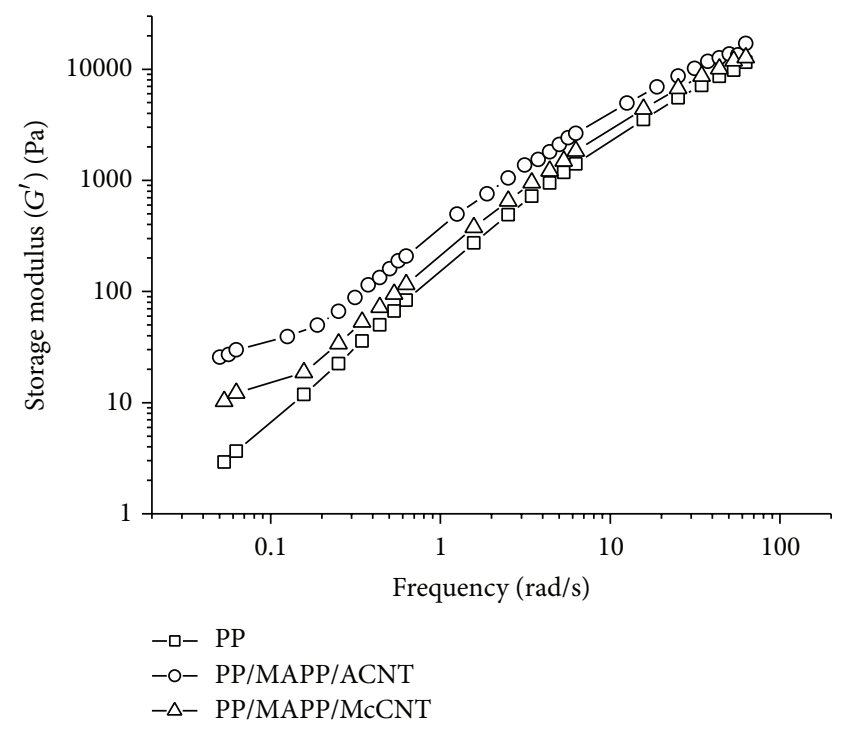

(a)

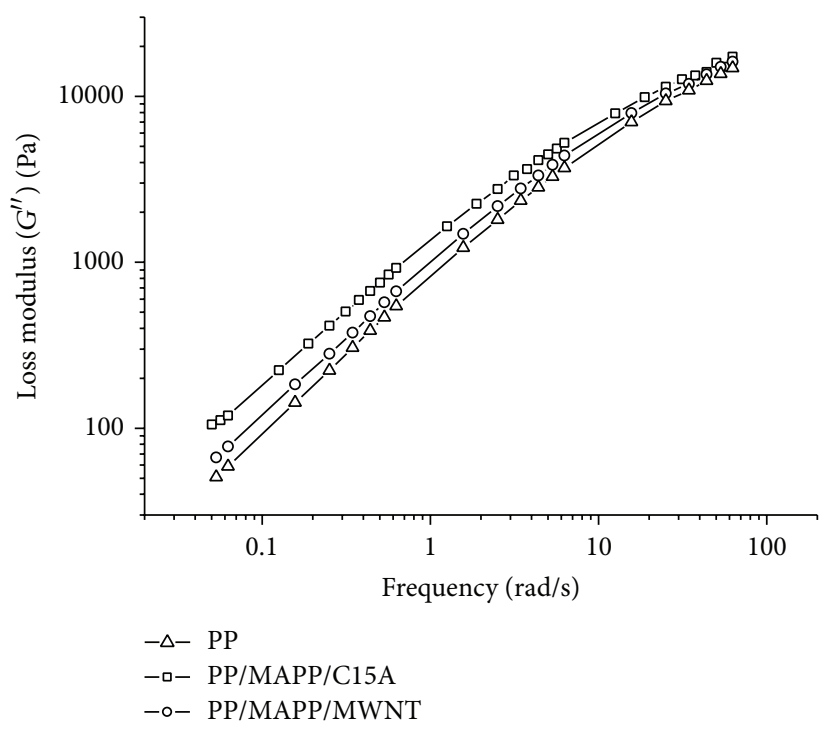

(b)

FIGURE 7: (a) Storage modulus versus frequency; (b) loss modulus versus frequency of PP and nanocomposites.

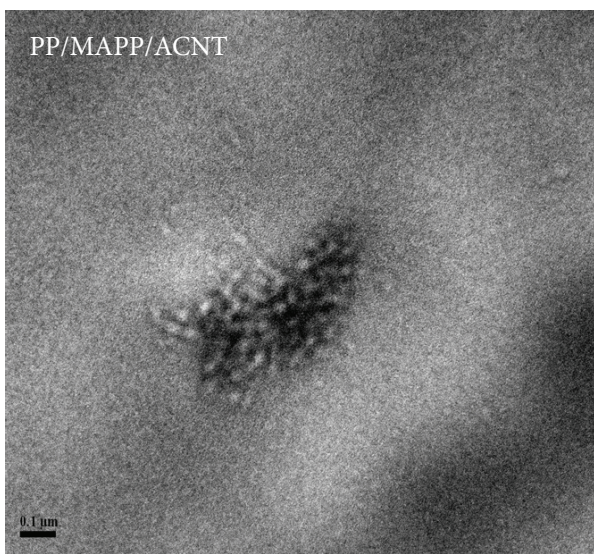

(a)

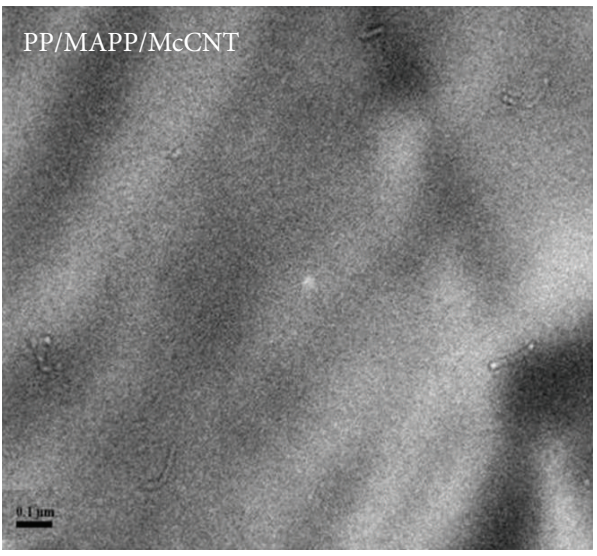

(b)

FIGURE 8: TEM images of PP nanocomposites.

the presence of the CNTs. Further, a decreased slope of $G^{\prime}$ and $G^{\prime \prime}$ of both the nanocomposites revealed the formation of interconnected and network like structure, resulting from nanotube-polymer interaction [14].

Further, from the analysis it was depicted that the extent of increase in $G^{\prime}$ of the PP and both the nanocomposites was higher than that of $G^{\prime \prime}$, revealing that the rheological properties of PP and both the nanocomposites can be sensitively explained by $G^{\prime}$ versus $\omega$ plot. Subsequently, it was depicted that the $G^{\prime}$ and $G^{\prime \prime}$ value of PP and its nanocomposites were higher as compared to neat $\mathrm{PP}$ in the low frequency region and this increasing effect was more pronounced in case of PP/MAPP/McCNT. Greater $G^{\prime}$ and $G^{\prime \prime}$ values of nanocomposites were ascribed to the formation of interconnected or networked structure, formed due to nanotube-polymer interaction. Subsequently, in the higher frequency region, both the nanocomposites exhibited the similar $G^{\prime}$ and $G^{\prime \prime}$ values as that of neat PP. This might be ascribed to the breakdown of interconnected network structure due to high level of shear force. However, the higher $G^{\prime}$ and $G^{\prime \prime}$ value of PP/MAPP/McCNT, as compared to $\mathrm{PP} / \mathrm{MAPP} / \mathrm{ACNT}$, confirmed the higher degree of interaction between McCNT and polymer matrix.

4.8. Transmission Electron Microscopy (TEM). TEM micrographs of the nanocomposites are reported in Figure 8. The micrographs showed the much uniform dispersion of the nanotubes in case of PP/MAPP/McCNT, revealing the better dispersibility and interaction of the McCNT in the polymer matrix. 


\section{Conclusions}

From the study we may conclude that the mechanical oxidation of the CNT via ball milling may be used as an effective method in order to modify the CNT and hence to reduce the Vander wall interaction between the tubes. Further, it was found that the mechanical oxidation method of CNT may be advantageous in several aspects as compared to acid oxidation method, by reducing the damage to the tubes. Hence, the intrinsic properties of the nanotubes may be intact. Further, since the CNTs are important as filler for polymer nanocomposite application, the polymer nanocomposites of the mechanically oxidized and acid oxidized CNT with polypropylene matrix were fabricated and investigated. The investigation revealed the higher nucleating ability of the McCNT as compared to ACNT. Also the highly uniform stress transfer ability in the PP/MAPP/McCNT was noticed as compared to PP/MAPP/ACNT. Also the rheological properties revealed the higher interaction between McCNT and polymer matrix as compared to ACNT.

Hence, it may be concluded that the mechanical oxidation of CNTs can be used as modification technique to improve the mechanical and microstructural properties of the polymer nanocomposites.

\section{Conflict of Interests}

The authors declare that there is no conflict of interests regarding the publication of this paper.

\section{References}

[1] Y.-S. Shim, B.-G. Min, and S.-J. Park, "Effects of functional grafting on viscoelastic and toughness behaviors of multi-walled carbon nanotubes-reinforced polypropylene nano-composites," Macromolecular Research, vol. 20, no. 5, pp. 540-543, 2012.

[2] K. Saeed and I. Khan, "Preparation and properties of singlewalled carbon nanotubes/poly(butylene terephthalate) nanocomposites," Iranian Polymer Journal, vol. 23, no. 1, pp. 53-58, 2014.

[3] X. Chen, J. Hu, L. Zhou, W. Li, Z. Yang, and Y. Wang, "Preparation and crystallization of carbon nanotube/maleic anhydridegrafted polypropylene composites," Journal of Materials Science \& Technology, vol. 24, no. 2, pp. 279-284, 2008.

[4] W. Xia, Y. Wang, R. Bergsträßer, S. Kundu, and M. Muhler, "Surface characterization of oxygen-functionalized multiwalled carbon nanotubes by high-resolution X-ray photoelectron spectroscopy and temperature-programmed desorption," Applied Surface Science, vol. 254, no. 1, pp. 247-250, 2007.

[5] H. Wang, W. Zhou, D. L. Ho et al., "Dispersing single-walled carbon nanotubes with surfactants: a small angle neutron scattering study," Nano Letters, vol. 4, no. 9, pp. 1789-1793, 2004.

[6] T. Kyotani, S. Nakazaki, W.-H. Xu, and A. Tomita, "Chemical modification of the inner walls of carbon nanotubes by $\mathrm{HNO}_{3}$ oxidation," Carbon, vol. 39, no. 5, pp. 782-785, 2001.

[7] N. V. Naseh, A. A. Khodadadi, Y. Mortazavi, O. A. Sahraei, F. Pourfayaz, and M. S. Sedghi, "Functionalization of carbon nanotubes using nitric acid oxidation and DBD plasma," International Journal of Chemical and Biological Engineering, vol. 2, no. 2, pp. 66-68, 2009.
[8] I. D. Rosca, F. Watari, M. Uo, and T. Akasaka, "Oxidation of multiwalled carbon nanotubes by nitric acid," Carbon, vol. 43, no. 15, pp. 3124-3131, 2005.

[9] A. B. González-Guerrero, E. Mendoza, E. Pellicer, F. Alsina, C. Fernández-Sánchez, and L. M. Lechuga, "Discriminating the carboxylicgroups from the total acidic sites in oxidized multi-wall carbon nanotubes by means of acid-base titration," Chemical Physics Letters, vol. 462, no. 4-6, pp. 256-259, 2008.

[10] M. M. Zamani, A. Fereidoon, and A. Sabet, "Multi-walled carbon nanotube-filled polypropylene nanocomposites: high velocity impact response and mechanical properties," Iranian Polymer Journal , vol. 21, no. 12, pp. 887-894, 2012.

[11] G. Z. Papageorgioul, M. Nerantzaki, I. Grigoriadou, D. G. Papageorgiou, K. Chrissafis, and D. Bikiaris, "Isotactic polypropylene/multi-walled carbon nanotube nanocomposites: the effect of modification of MWCNTs on mechanical properties and melt crystallization," Macromolecular Chemistry and Physics, vol. 214, no. 21, pp. 2415-2431, 2013.

[12] Y.-T. Shieh, G.-L. Liu, H.-H. Wu, and C.-C. Lee, "Effects of polarity and $\mathrm{pH}$ on the solubility of acid-treated carbon nanotubes in different media," Carbon, vol. 45, no. 9, pp. 18801890, 2007.

[13] C. Bower, A. Kleinhammes, Y. Wu, and O. Zhou, "Intercalation and partial exfoliation of single-walled carbon nanotubes by nitric acid," Chemical Physics Letters, vol. 288, no. 2-4, pp. 481486, 1998.

[14] J. Y. Kim, S. I. Han, and S. Hong, "Effect of modified carbon nanotube on the properties of aromatic polyester nanocomposites," Polymer, vol. 49, no. 15, pp. 3335-3345, 2008.

[15] M. S. Dresselhaus, G. Dresselhaus, R. Saito, and A. Jorio, "Raman spectroscopy of carbon nanotubes," Physics Reports, vol. 409, no. 2, pp. 47-99, 2005.

[16] C. Thomsen and S. Reich, "Double resonant Raman scattering in graphite," Physical Review Letters, vol. 85, no. 24, pp. 5214$5217,2000$.

[17] S. Osswald, E. Flahaut, H. Ye, and Y. Gogotsi, "Elimination of D-band in Raman spectra of double-wall carbon nanotubes by oxidation," Chemical Physics Letters, vol. 402, no. 4-6, pp. 422427, 2005.

[18] T. J. Simmons, J. Bult, D. P. Hashim, R. J. Linhardt, and P. M. Ajayan, "Noncovalent functionalization as an alternative to oxidative acid treatment of single wall carbon nanotubes with applications for polymer composites," ACS Nano, vol. 3, no. 4, pp. 865-870, 2009.

[19] N. Pierard, A. Fonseca, J.-F. Colomer et al., "Ball milling effect on the structure of single-wall carbon nanotubes," Carbon, vol. 42, no. 8-9, pp. 1691-1697, 2004.

[20] P. Liu, "Modifications of carbon nanotubes with polymers," European Polymer Journal, vol. 41, no. 11, pp. 2693-2703, 2005.

[21] S. K. Samal, S. K. Nayak, and S. Mohanty, "Banana/glass fiber-reinforced polypropylene hybrid composites: fabrication and performance evaluation," Polymer-Plastics Technology and Engineering, vol. 48, no. 4, pp. 397-414, 2009.

[22] H. Plaza, B. Reznik, M. Wilhelm, O. Arias, and A. Vargas, "Electrical, thermal, and mechanical characterization of poly(propylene)/carbon nanotube/clay hybrid composite material," Macromolecular Materials and Engineering, vol. 297, no. 5, pp. 474-480, 2012.

[23] V. Vladimirov, C. Betchev, A. Vassiliou, G. Papageorgiou, and D. Bikiaris, "Dynamic mechanical and morphological studies of isotactic polypropylene/fumed silica nanocomposites with 
enhanced gas barrier properties," Composites Science and Technology, vol. 66, no. 15, pp. 2935-2944, 2006.

[24] K. Prashantha, J. Soulestin, M. F. Lacrampe, P. Krawczak, G. Dupin, and M. Claes, "Masterbatch-based multi-walled carbon nanotube filled polypropylene nanocomposites: assessment of rheological and mechanical properties," Composites Science and Technology, vol. 69, no. 11-12, pp. 1756-1763, 2009.

[25] T. Kashiwagi, F. Du, J. F. Douglas, K. I. Winey, R. H. Harris Jr., and J. R. Shields, "Nanoparticle networks reduce the flammability of polymer nanocomposites," Nature Materials, vol. 4, no. 12, pp. 928-933, 2005. 

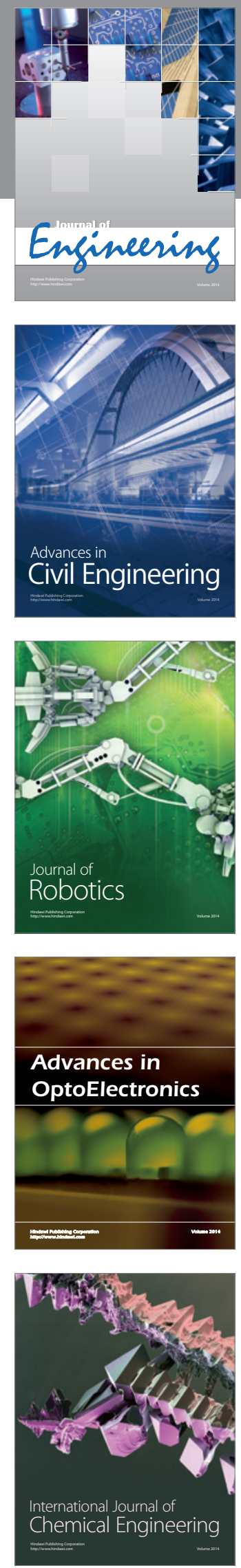

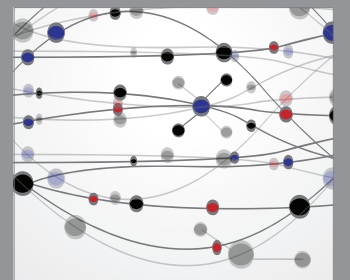

The Scientific World Journal
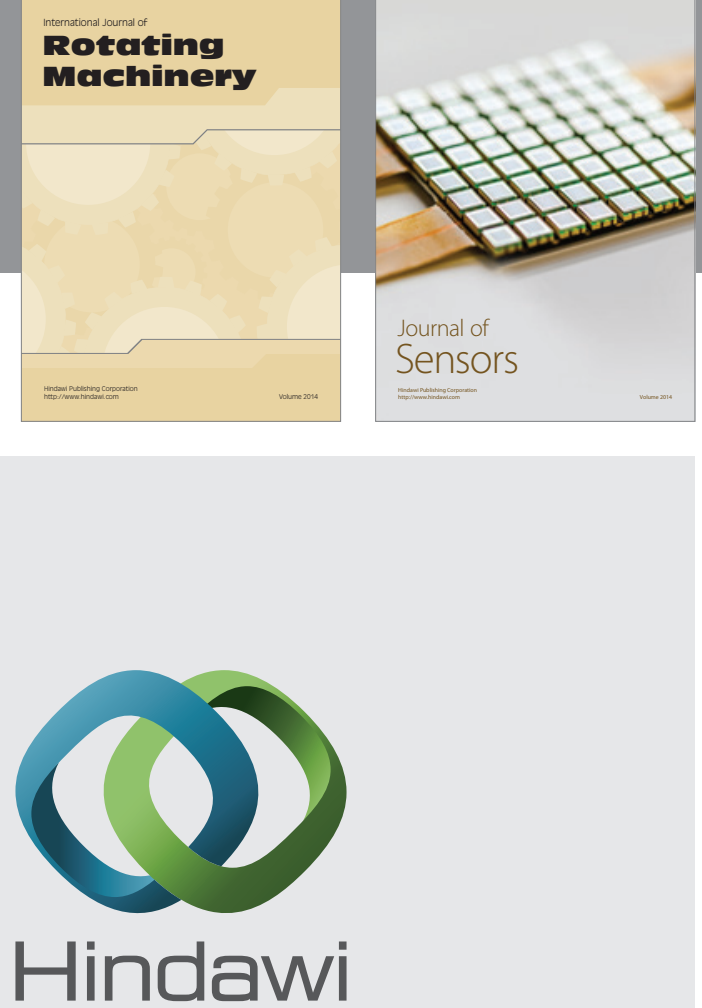

Submit your manuscripts at http://www.hindawi.com
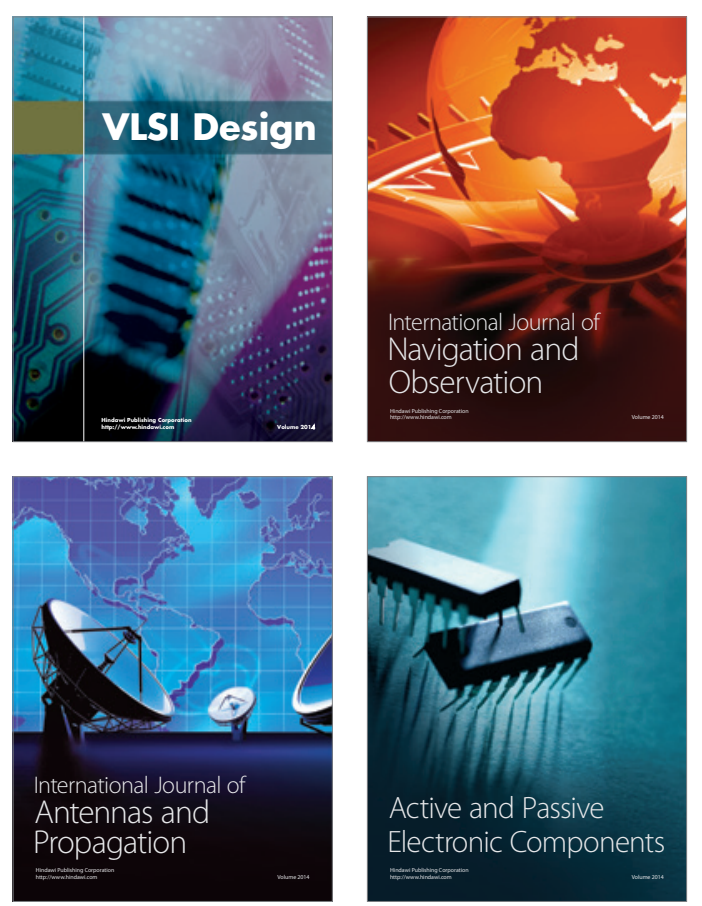
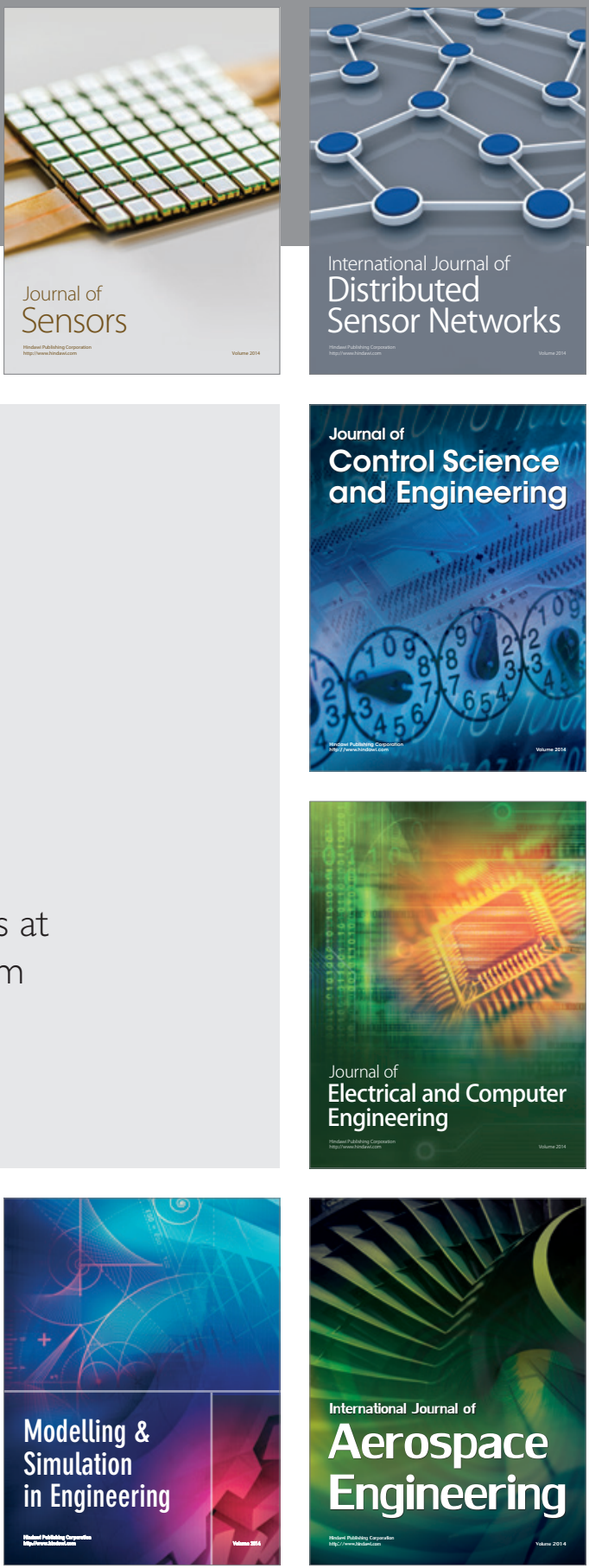

Journal of

Control Science

and Engineering
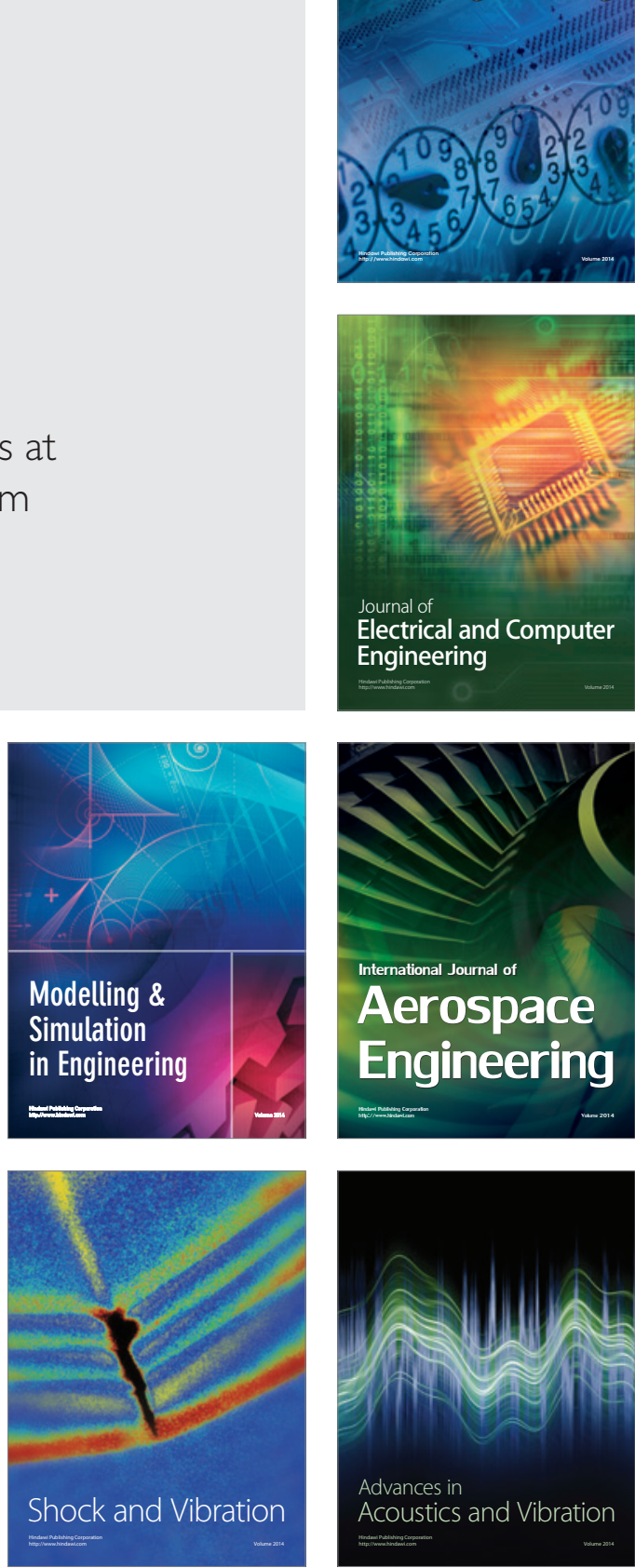